\title{
Hysteretic Behavior and Anisotropy Fields in the Magneto-Impedance
} Effect

\author{
M. Vázquez, J.P. Sinnecker and G.V. Kurlyandskaya \\ Instituto de Magnetismo Aplicado, UCM/RENFE, and Instituto de Ciencia de Materiales, CSIC, \\ P.O. Box 155, ES-28230 Las Rozas (Madrid), Spain
}

Kevwords: Magnetization Process, Magneto-Impedance, Amorphous and Nanocrystalline Materials

\begin{abstract}
An overview of the Magnetoimpedance effect (MI) in a series of magnetic materials with different geometries (wires, ribbons, tubes and thin films) and structures (crystalline, nanocrystalline and amorphous) is given. Two types of MI, namely single peak, SP, and two peak, TP, are discussed. The observation of both types of behaviours is general and depends on the amplitude and the frequency of a.c. current required to measure the impedance. A correlation between MI hysteresis and hysteresis loops is presented. The influence of the a.c. current frequency, magnetic anisotropies and irreversible magnetization processes upon the hysteretic MI effect is discussed
\end{abstract}

\section{Introduction.-}

The first and main consequence of the application of a d.c. magnetic field to a ferromagnetic material is the modification of its magnetic state via the magnetization process occurring by domain wall displacements and magnetization rotation within domains. In fact, the magnetic state at a given applied d.c. field can be characterized by the magnetic response to an additional a.c. magnetic excitation, which is represented by a differential permeability. This permeability is determined by a set of conditioning circumstances associated to the magnetic characteristics of the material itself.

In addition, the application of a magnetic field can first modify the transport properties via the magnetoresistance effect (scattering of electrons) which will not be treated here, but also the total impedance itself through an electromagnetic classical effect. Usually, the impedance is determined by the so-called four points technique which requires the sample to be submmitted to an electrical current, resulting in a given measurable voltage through it. The impedance is thus evaluated from the relationship between that voltage and the applied current.

Nevertheless, depending on the frequency of the exciting current one can obtain various results. In the limit of very low frequencies, the current just generates a circumferential magnetic field, which eventually acts superimposed to the above-mentioned d.c. applied field. As a consequence of the temporal variation of this circular field, changes of the circular component of magnetization are induced so resulting in an extra electromotive force at the ends of the sample according to the Faraday-Lenz law. Such changes of the circumferential component of magnetization are responsible for the so-called magneto-inductive effect ( $\mathrm{M}_{\phi} \& \mathrm{H}_{\phi}$ magnetic hysteresis loops) and for the Matteucci effect $\left(\mathrm{M}_{\phi} \& \mathrm{H}_{\mathrm{z}}\right.$ loops $)[1,2]$.

For higher frequencies, typically above $100 \mathrm{kHz}$, there is an effective reduction of the cross section through which the current is flowing owing to the generated eddy currents[3]. As a consequence of the skin effect, the current flows through a sheath close to the surface of the sample. In this case, only a radial fraction of the sample is involved in the high frequency magnetization process, and there is a correlation between the penetration depth and the magnetic permeability via the expression:

$$
\delta=\sqrt{\frac{1}{\pi \sigma v \mu}}
$$

where $\sigma$ is the electrical conductivity, $\nu$ the frequency of the current and $\mu$ the circular or transverse permeability

In turn, the total impedance, $Z$, is related to that penetration length according to 


$$
Z=R_{D C}\left(\frac{k a}{2}\right) \frac{J_{0}(k a)}{J_{1}(k a)}
$$

where $\mathbf{a}$ is the radius of wire, and $\mathbf{J}$ the Bessel functions. Consequently, upon application of a d.c. magnetic field, $\mathrm{H}$, the accompanying change of permeability finally results in an effective modification of the impedance.

The experimental techniques to perform magnetic and magnetoimpedive measurements are slighthly different for the various analysed samples so that, in the following we refer to indicated references for detailed description of experimental procedures. Just to avoid uncertainties, we will consider the present definition for the magneto-impedance ratio (GMI):

$$
\frac{\Delta Z}{Z}=\frac{\left[Z(H)-Z\left(H_{\max }\right)\right]}{Z\left(H_{\max }\right)} \times 100 \%
$$

where $Z(H)$ is the impedance measured at the dc field $H$ and $Z\left(H_{\max }\right)$ is the impedance measured at the maximum dc field.

Finally, at the limit of very high frequencies, above $100 \mathrm{MHz}$, the skin effect penetration depth can become comparable to atomic distance and resonance effects are observed, which is also out of the scope of the present work[4].

The giant magnetoimpedance, GMI, effect discovered recently in magnetic wires and other extremely soft magnetic materials is attracting much attention because of its technological applications[5-12]. Most of such applications deal with magnetic field sensor devices (i.e., current and position sensors) and magnetoelastic sensors (i.e., stress sensor and magnetostriction evaluation)[13]. Looking for these applications, it is required to determine which are the optimum conditions for which GMI is most effective. In particular, which is the optimum range of frequency to obtain large ratio GMI to noise, or the maximum field sensitivity for the specific intervale of field to be sensed. In this regard, it is quite important to know the differential transverse permeability response to applied d.c. fields, which is basically determined by general magnetic characteristics as domain structure and spatial distribution of magnetic anisotropy. In connection with such magnetic characteristics there are some effects as irreversibilities in the magnetization process or disaccommodation or magnetic after-effects which in principle represent an obstacle for a proper use of MI effect in technological applications[14,15].

In this work, we are collecting experimental results concerning the different behaviours observed for the MI as well as its irreversibility in various types of materials and analyse its correlation with the anisotropy fields and the hysteretic behavior of the corresponding loops for various kinds of materials as: water-quenched amorphous and nanocrystalline wires, policrystalline wires, glass-coated amorphous microwires, stress and field annealed nanocrystalline ribbons, electroplated tubes and processed sputtered thin films. This large variety of materials is considered to point out the generality of the presented results. In section 2 , we proceed with a general discussion about the various domain structures and magnetic anisotropies in the studied materials, while in section 3 we describe the two types of MI behaviors as associated to the transverse anisotropy field. In section 4, we will introduce the hysteretic behavior and correlate it with the transverse permeability in the above mentioned materials and introduce the irreversibility field. Finally, in section 5 a final discussion is presented on the correlation between the hysteresis in the magnetoimpedance and the magnetization process. The potential influence of that hysteretic behavior in technological devices is shortly commented.

Domain structure and a.c. circular magnetization process under bias d.c. magnetic field.-

According to Eq. 2 the circular permeability and its change with dc axial field is determining the actual amplitude of magnetoimpedance. This permeability will be determined by the particular domain structure of the material. Although we will consider also the cases of ribbons, tubes and films 
we restrict ourselves in this section to domain structrures of wires. A further analysis can be accordingly extended to the other geometries.

Owing to its cylindrical geometry we consider 3 major or ideal configurations for a wire with axial, circumferential and radial easy axis. A general information on domains in wires can be found elsewhere[16,17]. The first case is that of having an axial anisotropy which results ideally in a singledomain structure and square axial hysteresis loop with a given switching field at which the magnetization reverses between two stable configurations of domains. The presence of an applied d.c. reverse axial field nearly as large as the switching field has the effect of quasi-compensate the axial magnetic anisotropy. Magnetization feels quasi-free for an outstanding response under the action of incremental fields. If the easy axis is not fully axial but with some circular component (helical direction) such incremental field, will result in a noticeable circular permeability. As indicated in the scheme of Fig. 1a, the circumferential permeability peaks for a d.c. axial field close to the switching field. For quite small or very large d.c. fields, the result is nevertheless very modest. In short, there is an intrinsic irreversible and assymetric character for the circumferential permeability with the applied d.c. axial field. In this framework, we can introduce an "irreversibility field", $\mathbf{H}_{\text {irrv, }}$ corresponding to that switching field. Actually, this circular permeability appears from the existance of some helical component of the magnetization and is essentially irreversible. Considering the magneto-impedance effect, a peak when increasing the axial bias field is expected when the switching field is reached.

The second case is when the easy axis of intrinsecal anisotropy is circumferential and the domain structure is a bamboo-like structure as indicated in Fig. lb. Now the dc axial field plays a role to balance the circumferential anisotropy so that, a large peak for the circumferential permeability is obtained just when the applied field reaches the anisotropy field, $\mathrm{H}=\mathrm{H}_{\mathrm{k}}$. This permeability behaves nearly reversibly with increasing and decreasing d.c. field but with some small hysteretic component inherent to the magnetization rotation. Accordingly, the MI ratio should exhibit a maximum value for a bias field equaling the circular anisotropy field.

Finally, in the case of having strong enough radial anisotropy, a radial network of domains is

(a)

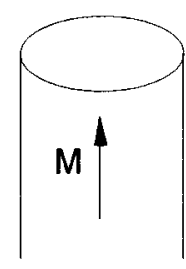

(b)

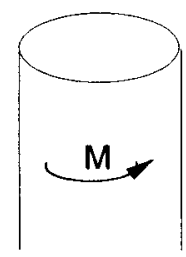

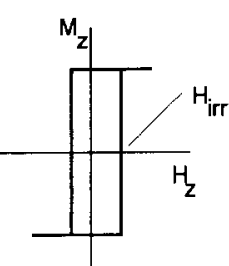

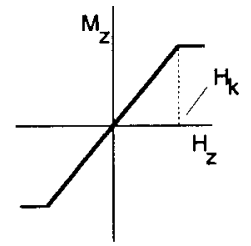

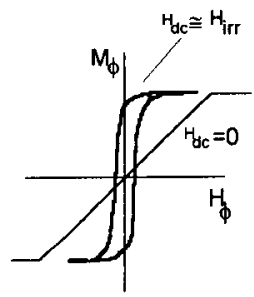

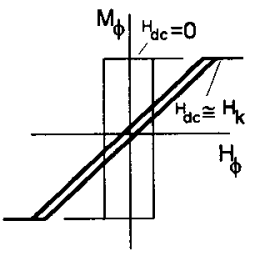

Figure 1: Schematic view of the effect of a dc axial applied field on the axial and circular magnetization curves for wires with (a) axial and (b) circular easy magnetization directions.

expected. Now, the bias field balances the radial anisotropy field, and a large circumferential permeability is expectable when, as in the first case, there is some circular component now for the radial anisotropy. Both kinds of behaviors in the MI are now expected: peaks for the irreversible field and for the anisotropy field.

\section{The two types of magnetoimpedance behavior.-}

Provided that the amplitude and frequency for the current flowing along the sample are fixed, it is currently spoken of two types of magnetoimpedance behaviors upon increasing the d.c. axial 
magnetic field[10]. In the following we will label them as: i) Single-peak, SP, behavior where a continous decrease of the magneto-impedance ratio is observed, and ii) Two-peaks, TP, behavior where the maximum magneto-impedance ratio is shifted from zero up to a given applied field followed by the monotonic decrease. Moreover, the field at which this maximum is observed has been currently ascribed or correlated to the transverse magnetic anisotropy field. The occurrence of these two behaviors, apparently different, has been discussed recently[18]

We will explore here the consequences of the following reasoning: as the maximum MI ratio is observed for a given d.c. applied field when there is a maximum change of circumferential permeability (i.e., the a.c. permeability reaches a maximum), the first mentioned behavior would correspond to the case where the easy magnetization direction for the magnetic anisotropy is nearly parallel to wire's axis. But even in this case some finite longitudinal field is required to roughly attain the magnetization saturation. Such a finite field is ascribed to the inherent (although maybe quite small) irreversible component of the axial magnetization process which has been described in the preceeding section. Consequently, in this case the maximum MI ratio would correspond to that finite field at which the irreversible magnetization process is completed. This argumentation predicts that SP behavior is a particular case of the TP if we zoom in detail enough into the very small field region. So, in the following we are considering two axial fields determining the maximum $\mathrm{MI}$ ratios namely, the axial field equaling the transverse anisotropy field, $\mathrm{H}_{k}$, and the so-labelled irreversibility field, $\mathrm{H}_{\mathrm{irr}}$, corresponding to the field ascribable to the completion of the irreversibilites of the axial magnetization process.

In order to check that, we first consider the probably most traditional magnetoimpedive material as is a negative-vanishing magnetostriction Co base amorphous wire. As reported in early papers on GMI, this outstandingly soft material can be considered in its as-cast state as an example for the single-peak behavior. So, looking into detail, we searched for the two-peaks behavior[19]. Both types of results are given in Fig. $2 a$ and $2 b$ respectively where it is unveiled the importance of the plotting scale to observe the second one.

On the other hand, the observation of these SP or TP behaviors depends upon the frequency and amplitude of the a.c. current[20,5]. This is clearly shown in the figures $3 \mathrm{a}$ and $3 \mathrm{~b}$. In Fig. $3 \mathrm{a}$, the frequency dependence of the magnetoimpedance ratio is shown for a policrystalline permalloy wire after being submmitted to a particular treatment. While for relatively low frequency, a SP like behavior is observed, TP characteristics appear at the range of higher frequencies. As observed, not only the value of the maximum MI ratio changes with frequency but also the applied field at which the maximum is observed shifts towards higher field with increasing frequency. This shifting has been recently studied in detail in glass coated amorphous microwires[21]. The shifting of the peaks with increasing frequency towards higher bias fields in

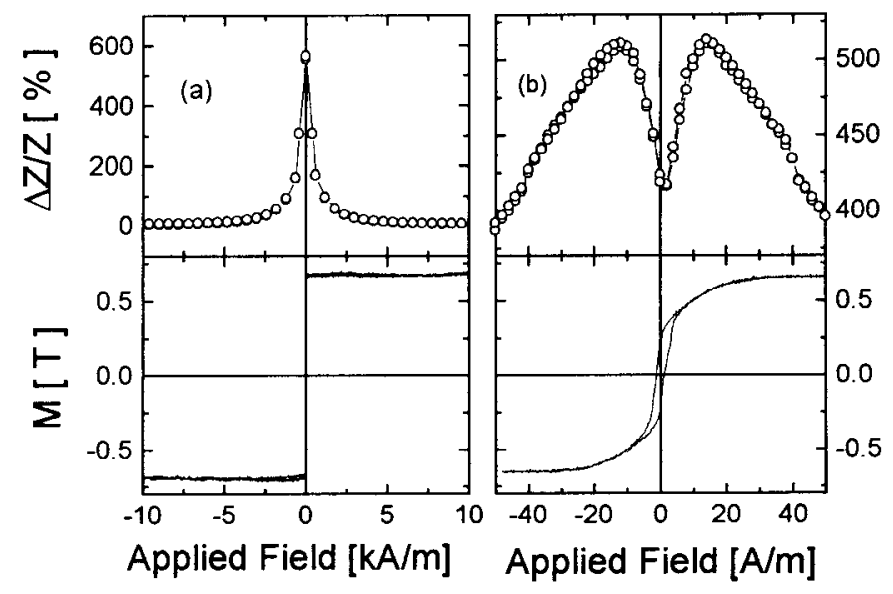

Figure 2 : Magnetoimpedance and axial hysteresis loops of as cast $\mathrm{CO}_{68.15} \mathrm{Fe}_{4.35} \mathrm{Si}_{12.55} \mathrm{~B}_{15}$ amorphous wires showing a Single Peak (a) and a Two Peak (b) behavior. 
the TP behavior is ascribed to the increasing average magnetoelasic anisotropy in the region involved in the skin-effect penetration depth[22]. In Fig. 3b, a large modification of the MI aspects with the amplitude of the a.c. current is shown for a colddrawn and subsequently stress-annealed $\mathrm{Co}$ rich amorphous wire[23]. In fact, the picture evolves from SP to TP when decreasing the amplitude. The TP behavior is actually a consequence of not reaching the circular coercivity. In the case of the sample of the Fig. $3 \mathrm{~b}$, the domain structure is purely circumferential. These modifications are discussed in
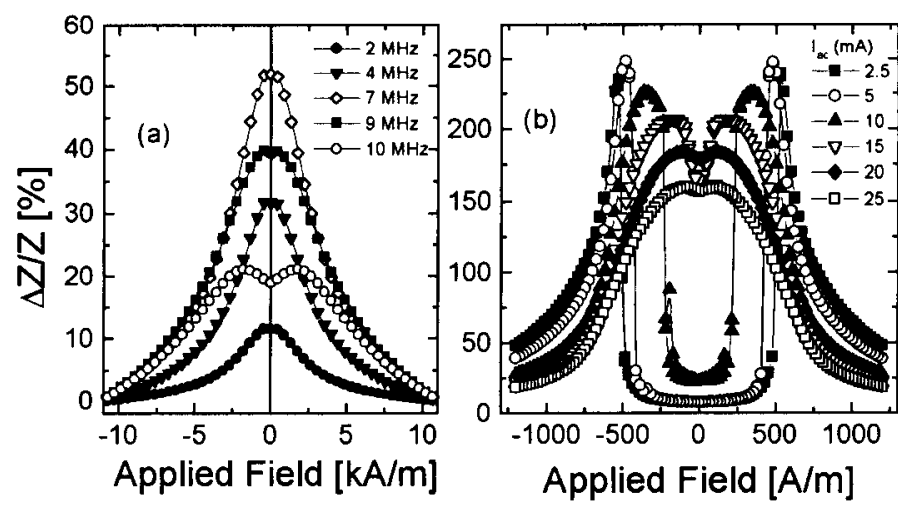

Figure 3 : (a) Frequency dependence of the magnetoimpedance measured in Carpenter HyMu $80^{8}$ alloy $\left(\mathrm{Ni}_{80} \mathrm{Mo}_{4.2} \mathrm{Fe}_{\text {bal }}\right.$ permalloy). The sample is obtained after a cold-drawn process is carried out in the as-cast wire. (b) Magnetoimpedance dependence with the amplitude of the a.c. current for a cold-drawn and subsequently stress-annealed Co rich amorphous wire.

\section{a recent work as arising from}

the particular characteristics of the transverse permeability at the working conditions. In short, and as far as the appearance of SP and TP behaviors with frequency and and amplitude, the drift of the field for the maximum MI with frequency would indicate the increase of the anisotropy field ascribeable to the region containing the penetration depth. In turn, the amplitude of the current determines a critical value for which the coercivity is reached in the circular magnetization process under the biasing effect of the axial d.c. field[1]

\section{Hysteretic Magneto-Impedance.-}

The appearance of some hysteretic behavior in the magnetoimpedance effect has been reported in some previous works[24-28,5]. But no general or systematic study has been performed. Nevertheless, this is an undesirable effect for most pourposes especially in technological applications as for instance in sensor devices. Consequently, the study to determine under which conditions it appears seems very justified.

As mentioned above, there has been a few reports mentioning the GMI hysteresis. But which is its origin?. We know that the origin of the MI phenomenon is related to skin effect penetration depth and its modification with an applied dc field through the change of the circumferential permeability. But, as we have discussed in the preceeding section, that change in the permeability must follow the normal hysteretic behavior accompanying the magnetization processes. It means that an apparent hysteresis for the GMI should appear in a case when there is large hysteretic behavior of the magnetization process. This point is actually difficult to observe since precisely, the appearance of GMI requires very soft magnetic behavior and quite large values of permeability which in principle is in contradiction with a large hysteretic behavior.

An experiment was performed to observe large hysteretic GMI[29]. It was done in an Fe base amorphous wire with large and positive magnetostriction. In its as-cast state it exhibits two main regions with axial (core) and radial (sheath) easy magnetization directions so that, practically no $\mathrm{MI}$ effect is observed. A thermal treatment was performed in order to relaxe the radial anisotropy and 
increase the circumferential permeability[30]. Moreover, partial crystallization was even achieved. The details of the procedure can be found elsewhere, and both the low frequency axial hysteresis loop and the magneto-impedance effect can be observed in the Fig. 4. The two following points should be underlined: i) First of all is the fact of observing relatively large (altough much smaller than for nearly zeromagnetostriction alloys) $\mathrm{MI}$ in this sample with large magnetostriction, and ii) There is quite a large magnetoimpedance hysteresis as was predictable. Looking at the MI effect in detail we can observe that the peak that actually gives rise to its hysteresis appears at the d.c. field corresponding to the coercivity of the hysteresis loop, which clearly support the previous assumption about the correlation of an irreversibility field, $\mathrm{H}_{\text {irr }}$.

That case is really a very peculiar one, but we wanted also to rediscover the hysteretic MI in more common magnetoimpedive materials but with differentiated magnetization processes. The first case we have analysed is that one of a sample with a well defined transverse anisotropy field but also exhibiting irreversibilities. In figure 5 , the magnetoimpedance effect and the axial hysteresis loop are shown for a material with improved technological capabilities as a nearly non-magnetostrictive $\mathrm{Co}$ base glass-coated amorphous microwire[31,13]. As observed, there is a clear transverse anisotropy of magnetoelastic origin determined by the internal stresses frozen in during the fabrication process. Besides this characteristic, there is also an hysteretic component in the magnetization process. In this case, the MI shows the TP behavior but if analysed in detail, a small, but detectable and repetitive, hysteresis appears between the two peaks. This hysteresis can be certainly adscribed to the hysteresis of the loop, and here, the irreversibility field, $H_{\text {irvv }}$, roughly equals the transverse anisotropy field, $H_{k}$.

The next step has been to find a material with well defined transverse anisotropy exhibiting also hysteresis but with $\mathrm{H}_{k}$ well separated from $\mathrm{H}_{\text {irr. }}$. This is the case of the Fe base amorphous alloy (now with ribbon shape) with particular composition so that a fine nanocrystalline microstructure is stable. The particular treatment was to devitrify the amorphous alloy under an applied tensile stress. More details about how this hysteresis loop and the particular domain structure are determined are given elsewhere. The results are plotted in the Fig. 6 where both the axial hysteresis loop and the hysteretic magnetoimpedance behavior are given

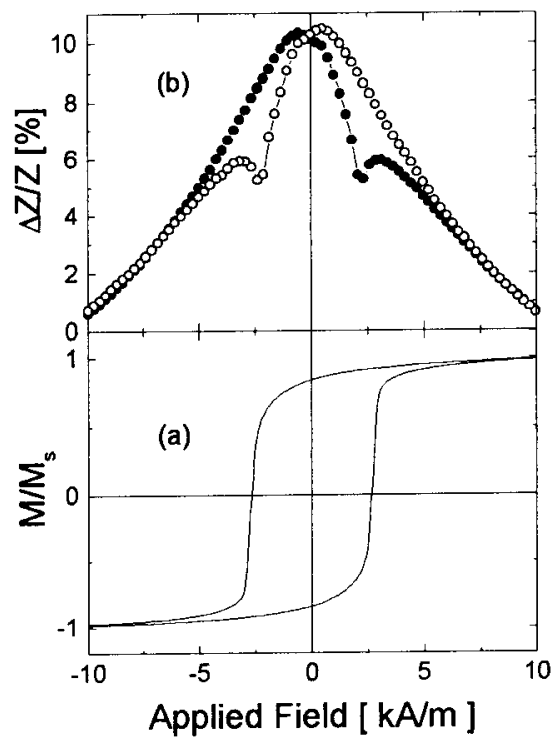

Figure 4: Magnetoimpedance and hysteresis loops for a $\mathrm{Fe}_{77.5} \mathrm{Si}_{7.5} \mathrm{~B}_{15}$ current annealed wire.

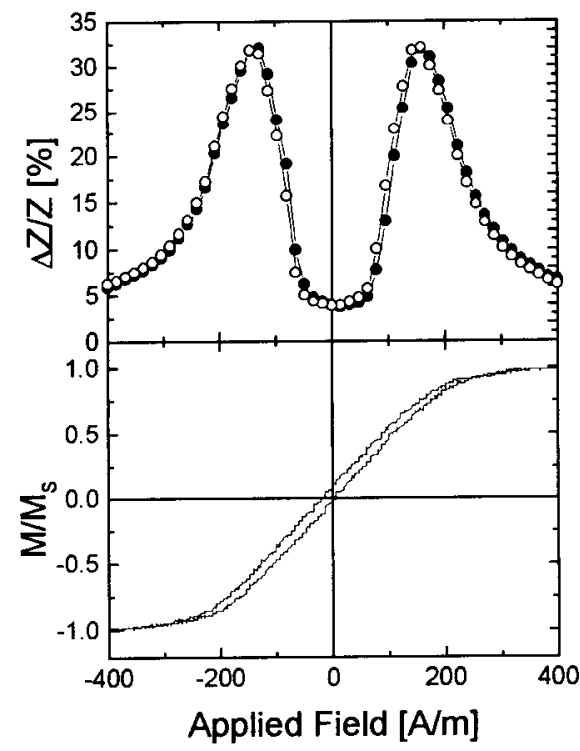

Figure 5: Magnetoimpedance and hysteresis loop measured in glass covered $\mathrm{Co}_{68.5} \mathrm{Mn}_{6.5} \mathrm{Si}_{10} \mathrm{~B}_{15}$ microwire. A slightly hysteretical behavior in the MI curve can be observed. 
and compared[32]. A correlation between irreversibility and anisotropy fields clearly followed.

A novel typed of materials, electroplated magnetic tubes, has been recently reported to show MI effect[33]. Additonal experimental work on these materials is being extensively followed by us in a number of novel materials as: $\mathrm{Co}$ base (Fig. 7)[34] and NiFe (Fig.8)[35] soft tubes electrodeposited on cupper wire. Fig. 7 corresponds to an as obtained sample, and from the combined evaluation of hysteresis loop and observed domain pattern, a radial magnetic anisotropy is
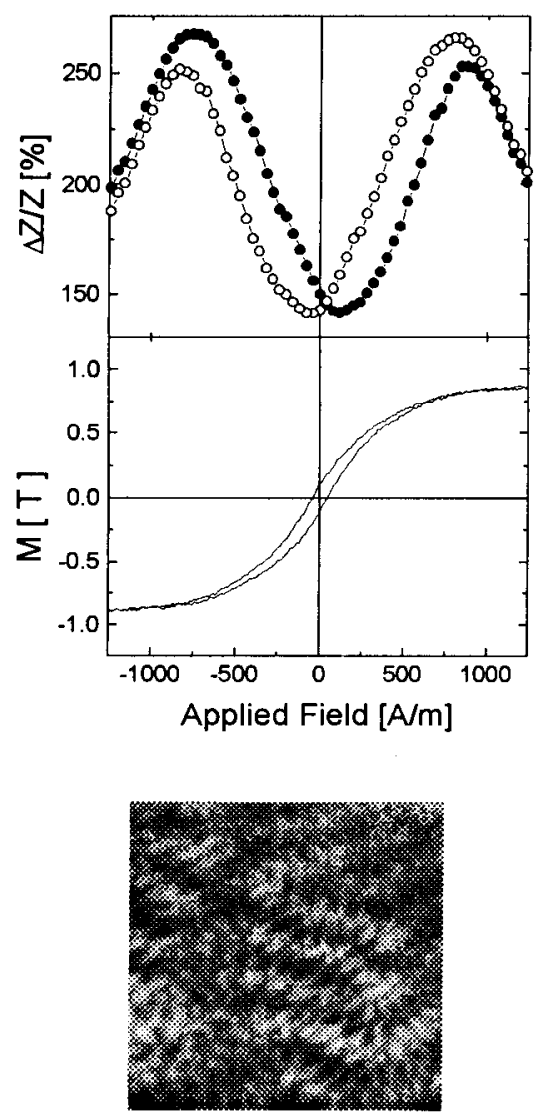

Figure 7: Hysteresis loops, magnetoimpedance and MFM microscopy surface domain structure image obtained for a $\mathrm{Cu}$ wire with a $9 \mu \mathrm{m} \mathrm{CoP}$.
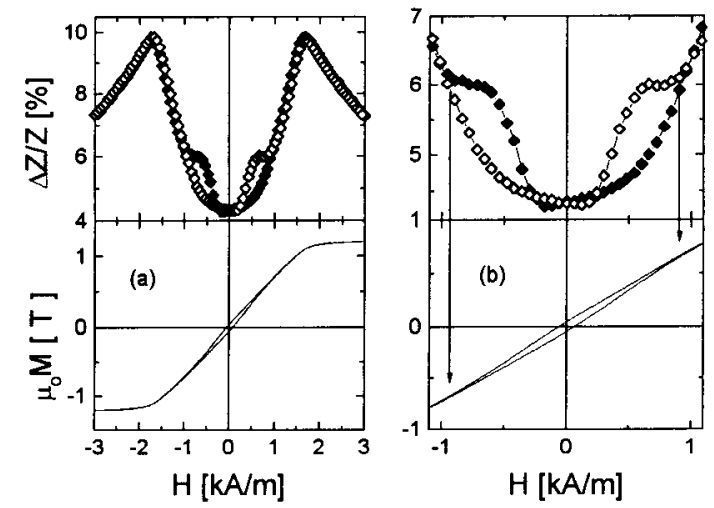

Figure 6 : Magnetoimpedance and hysteresis loops in stress annealed $\mathrm{Fe}_{73.5} \mathrm{Cu}_{1} \mathrm{Nb}_{3} \mathrm{Si}_{13.5} \mathrm{~B}_{9}$ ribbons.

deduced. Looking at the MI, a hysteresis is observed with roughly $\mathrm{H}_{\mathrm{irrv}}=\mathrm{H}_{\mathrm{k}}$. This behavior somehow remembers us to that one shown in Fig. 4 for the treated $\mathrm{Fe}$ base wire. The presence in both cases of a radial component of anisotropy could be associated to

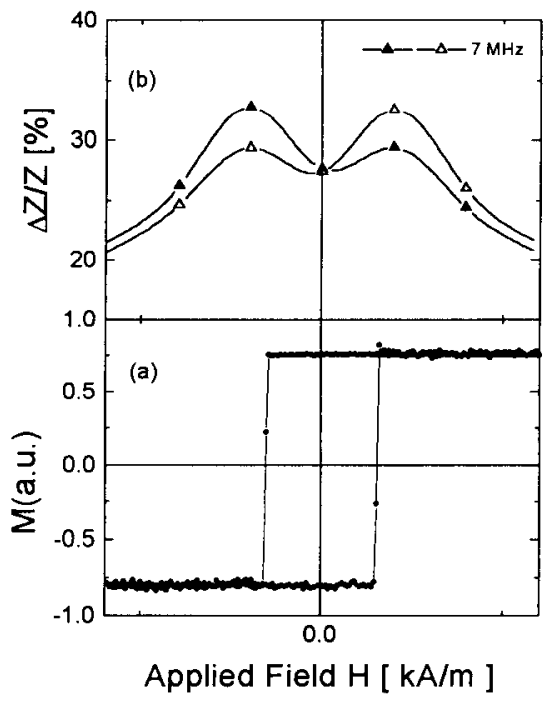

Figure 8: (a)Hysteresis Loops $\begin{aligned} & \text { and } \\ & \text { magnetoimpedance in } \mathrm{Cu} \text { wires }\end{aligned}$ with an
eletrodeposited layer of NiFe.

the large observed MI hysteresis. In the case of Fig. 8, the sample was submmitted to an axial-field annealing 


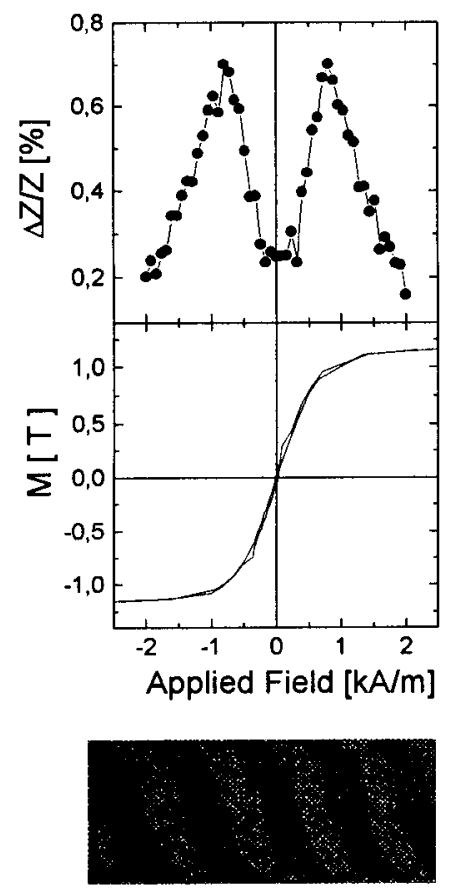

Figure 9 : Hysteresis loops magnetoimpedance and Kerr effect surface domain structre obtained for a $\mathrm{CoFeB}$ sputtered thin film. so inducing an axial magnetic anisotropy as can be deduced from the axial hysteresis loop. It is remarkable the appearance of hysteresis in the MI also for this type if anisotropy as was discussed in section 2. This hysteresis is anyway less significant than that shown in Fig. 7.

\section{Final Analysis and Discussion}

A clear correlation between domain structure, magnetization process and magneto-impedance behavior has been proved experimentally along the preceeding sections. A final example is given in Fig. 9 for a sputtered thin film submmitted to a thermal treatment under a.c. transverse field[36]. It exhibits a well defined anisotropy and TP magnetoimpedance behavior.

A comparison between domains structure, magnetization process and MI has been reported previously by several authors $[18,25,37,38]$. Depending on the orientation of the easy magnetization direction, different MI characteristics are nevertheless observed. Considering the equilibrium condition for the Zeeman and anisotropy energy terms, we introduce a general expression for the circumferential permeability as:

$$
\mu=\alpha M_{s}\left(\left(H-\alpha H_{k}\right)^{-1}+\left(H-\beta H_{i r v v}\right)^{-1}\right)
$$

where $\alpha$ is a parameter approaching 1 in the case of small amplitudes for the transverse anisotropy and a.c. current, while $\beta$ takes into account the particular pinning centers structure. According to this general expression local maximum values are expectable when the applied field reaches either $\mathrm{H}_{\mathrm{k}}$ or $\mathrm{H}_{\mathrm{inv}}$. Nevertheless an additional complexity must be taken into account arising from the non-homogeneous distribution of magnetic anisotropies[13]. For instance, in the case of amorphous wires, a radial distribution for the transverse permeability is expected (see Fig. 10a) coming from the intrinsecal distribution of internal stresses. Such distribution can be smoothed (Fig.

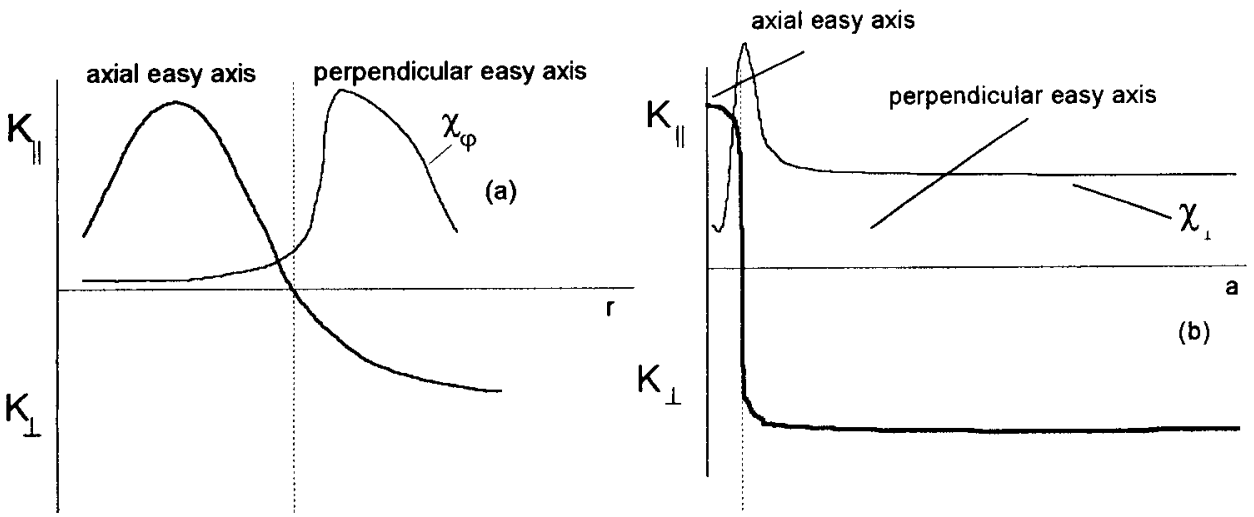

Figure 10: Schematic view of the magnetic anisotropy constant $K$ profile and trasnversal susceptibility in (a) amorphous as cast wires and (b) nanocrystalline annealed ribbons with transversal anisotropy. 


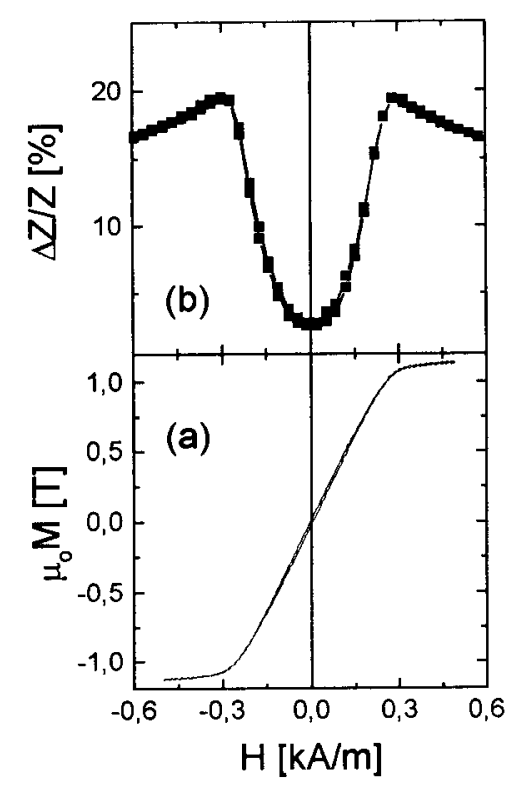

Figure 11 : (a) Hysteresis and (b) magnetoimpedance measured in a field annealed nanocrystalline ribbon. 10b) in the cases when the samples are submmitted to adequate treatments and in particular to magnetic field annealing. This is shown in Fig. 11 for a ribbon which has been submmitted to a transverse field annealing while devitrifying from amorphous structure into a nanocrystalline structure embedded in a residual amorphous matrix[39].

\section{Conclusions.}

As a general conclusion, we present a systematic study in different samples showing the correlation between magnetization process, domain structure and magnetoimpedance ratio.

We have also established and characterised the existence of SP and TP behaviors for any sample which can be observed depending on the frequency and amplitude of the a.c. current flowing along the samples. The observation of hysteresis in the magnetoimpedance is on the other hand clearly correlated to the irreversible magnetization processes.

As far as the use of this MI phenomenon in samples for sensing elements in sensor devices is concerned, a SP behavior with vanishing hysteresis is in general desirable. In order to have this particular behavior, we can finally conclude that the easy magnetization direction should be essentially parallel to the transverse field created by the a.c. current, and that the strength of such a transverse anisotropy must be as small and homogeneous as possible.

\section{Acknowledgments}

The authors are deeply endebted to many researchers at the Instituto de Magnetismo Aplicado for graciously supplying experimental material presented in this repport. In particular thanks are given to highly qualified Ph.D. students D. Garcia, J.L. Muñoz, J.M. García-Beneytez, J.J. Freijo and J.M. Garcia, to Dr. A.Asenjo and Dr. A. Mendez (ABB, Raleigh, U.S.A), to Dr. A.Garcia Arribas(Universidad de Oviedo) and to Dr. D.-X. Chen and Prof. A. Hernando. Dr. J.P. Sinnecker wishes to thank the Brasilian agency FAPESP and Dr. G. Kurlyandskaya to the Spanish Ministery of Education for finantial support during their stays at the IMA. The work has been supported by the Spanish CICYT under project MAT/95-0273.

\section{References}

[1] J. Velázquez, M. Vázquez, D.-X. Chen and A. Hernando, Phys. Rev. B 50 (1994), p. 16737.

[2] K. Mohri, T. Kohzawa, H. Yoshida and L.V. Panina, IEEE Trans. Magn. 28 (1992), p. 3150.

[3] L.D. Landau, E.M. E.M. Lifshift and L.P.Pitaevskii, Electrodynamics of Contimuous Media (Butterworth-Heinemann, Washington DC, 1995), p. 210.

[4] P. Ciureanu, M. Britel,, D. Menard, C. Akyel, A. Yelon, M. Rouabhi, R.W. Cochrane, P. Rudkowski and J.O. Strom-Olsen, J. Appl. Phys. (in press).

[5] L.V. Panina, K. Mohri, T. Uchiyama and M. Noda, IEEE Trans. Magn. 31 (1995), p. 1249.

[6] R.S. Beach and A.E. Berkowitz, Appl. Phys. Letters, 64 (1994), p. 3652.

[7] K.V. Rao, F.B. Humphrey and J.L. Costa-Kramër, J. Appl. Phys. 76 (1994), p. 6204.

[8] M. Knobel, M.L. Sánchez, J. Velázquez and M. Vázquez, J. Phys.: Condens. Matter. 7 (1995), p. L-115. 
[9] F.L.A. Machado, C.S. Martins and S.M. Rezende, Phys. Rev. B51 (1995), p. 3926.

[10] R.L. Sommer and C.L. Chien, Appl. Phys. Letter 67 (1995), p. 3346.

[11] M. Tejedor, B. hernando, M.L. Sánchez and A. García-Arribas. J. Magn. Magn. Mat. 157/158 (1996), p. 141.

[12] W. Ku, F. Ge and J. Zhu, J. Phys. D : Appl. Phys. 30 (1997), p.1842.

[13] M. Vázquez, M. Knobel, M.L. Sánchez, R. Valenzuela and A.P. Zhukov, Sensors \& Actuators A 59 (1997), p. 20.

[14] L.V. Panina and K. Mohri, J. Magn. Magn. Mat. 157/158 (1996), p. 137.

[15] M. Knobel, M.L. Sartorelli and J.P. Sinnecker, Phys. Rev. B 55 (1997), p. 3362.

[16] M. Vázquez and D.-X. Chen, IEEE Trans. Magn. 31 (1995), p. 1229.

[17] P.T. Squire, D. Atkinson, M.R.J. Gibbs and S. Atalay, J. Magn. Magn. Mat. 132 (1994), p. 10

[18] D.-X. Chen, J.L. Muñoz, M. Vázquez and A. Hernando, Phys. Rev. B 57 (1998) (in press).

[19] M.L. Sánchez, M. Knobel and D.-X. Chen, Nanostructured and Non-crystalline Materials, Ed. M. Vázquez and A. Hernando (World Scientific, Singapore, 1995), p. 592.

[20] V.E. Makhotin, B.P. Shurukhin, V.A. Lopatin, P.Yu. Marchukov and Yu.K. Levin. Sensors \& Actuators A, 25-27 (1991), p. 759.

[21] M. Vázquez, A.P. Zhukov, P. Aragoneses, J. Arcas, J.M. Garcia-Beneytez, P. Marin and A Hernando. IEEE Trans. Magn. 34 (1998) (in press).

[22] M. Vázquez, J.M. García-Beneytez, J.P. Sinnecker and L. Li, J. Appl. Phys. 83 (1998) (in press).

[23] J.J. Freijo, V.R. Ramanan, M. Vázquez and A. Mendez, (to be published)

[24] R.L. Sommer and C.L. Chien, J. Appl. Phys. 79 (1996), p. 5139.

[25] D. Menard, D. Frankland, P. Ciureanu, A. Yelon, M. Rouabhi, R.W. Cochrane, H. Chiriac and T.A. Ovari, J. Appl. Phys. (in press)

[26] R.L. Sommer and C.L. Chien, Appl. Phys. Letter 67 (1995), p. 857.

[27] K.C. Mendes, F.L.A. Machado, L.G. Pereira,S.M. Rezende, F.C. Montenegro, M.V. Altoé and F.P. Missell, J. Appl. Phys. 79 (1996), p. 6555.

[28] F.L.A. machado, B. Lopes da Silva and E. Monterroyos, J. Appl. Phys. 73 (1993), p. 6387.

[29] J.P. Sinecker, P. Tiberto, G.V. Kurdlyandskaya, E.H.C. Sinnecker, M. Vázquez and A. Hernando. J. Appl. Phys. (to be published)

[30] Y. Takemura, H. Tokuda, K. Komatsu, S. Masuda, T. Yamada, K. Kakuno and K. Saito. IEEE Trans. Magn. 32 (1996), p. 4947.

[31] M. Vázquez and A.P. Zhukov, J. Magn. Magn. Mat. 160 (1996), p. 223.

[32] M. Vázquez, G.V. Kurlyandskaya, J.L. Muñoz, A. Hernando, N.V. Dimitrieva, V.A. Lukshina and A.P. Potapov. J. Magn. Magn. Mat. (in press).

[33] R.S. Beach, N. Smith, C.L. Platt, F. Jeffers and A.E. Berkowitz. Appl. Phys. Letter 68 (1996), p. 2753.

[34] J.P. Sinnecker, A. Garcia-Arribas, J.M. García, A. Asenjo and M. Vázquez (to be published).

[35] D. Garcia, G.Kurlyandskaya, M. Vázquez, F.I. Toth and L.K. Varga, (to be published).

[36] D. Garcia, J.L. Muñoz, G. Kurlyandskaya, M. Vázquez, M. Ali and M.R.J. Gibbs, IEEE Trans. Magn. (in press).

[37] A.D. Santos, L.G.C. Melo, C.S. Martins, F.P. Missell, Y. Souche, F.L. Machado and S.M. Rezende, J. Magn. Magn. Mat.

[38] D. Atkinson and P.T. Squire, J. Appl. Phys. (in press).

[39] G.V. Kurlyandskaya, J.M. García-Beneytez, M. Vázquez, J.P. Sinnecker, V.A. Lukshina and A.P. Potapov, J. Appl. Phys. (in press). 